\title{
Moscow University Maths Department for Schoolchildren
}

Alexander V. Begunts and Anton E. Pankratiev (Lomonosov Moscow State University, Russia)

\section{Minor Department of Mechanics and Mathematics of Lomonosov Moscow State University (Malyi Mechmath)}

The Minor Department of Mechanics and Mathematics ("Malyi Mechmath" - "Junior Maths Department") comprises study groups for motivated schoolchildren keen on mathematics. It is hosted by the Department of Mechanics and Mathematics of Lomonosov Moscow State University and was founded in the late 1970s through the initiative of some young mathematicians (and later professors) S.B. Gashkov, I.N. Sergeev and Ya.V. Tatarinov, who managed to unite independent evening classes for residents and distance learning courses. Now, the resident classes pertain to the Evening Division and the distance learning courses have been reorganised into the Correspondence Division of Minor Mechmath.

Originally, the Correspondence Division of Minor Mechmath worked via the postal system. Students received printed material containing theoretical background on a subject, examples of problem solutions and a set of problems to be solved on their own. Nowadays, this form of studying employs modern educational technology and attracts schoolchildren from all over the world, including countries such as Germany and the United States.

Resident classes are held on a weekly basis (on Saturdays) from late September to the middle of May. They are oriented at schoolchildren aged from 8 to 18 and involve over 3,000 attendees of various mathematical skill levels. Attendance is free of charge and there are no entrance exams, nor are there any other constraints for participation in the classes. Such massive involvement and availability is due to the enthusiastic and thorough work of undergraduates and postgraduates of MSU under the supervision of highly experienced tutors. The basic principles of Minor Mechmath are an individual approach for every attendee, an activation of their creativity, a stimulation of their interest in mathematics and an encouragement of independent thinking.

Attendees of the classes are offered interesting, nonstandard problems in various fields of elementary mathematics. They learn to conduct rigorous logical reasoning and come to understand and feel the beauty and harmony of mathematics.

The lesson topics are quite diverse and they have little in common with the standard mathematical programmes of secondary schools. Subjects of the lessons include parity, divisibility, mathematical induction, backward reasoning, the principle of the extreme element, colourings, cuts and tessellations, graphs, combinatorics, compass and ruler geometry, decomposition-equal figures, motions of the plane, metrics, elementary topology, probability, the pigeonhole principle, inequalities, centre of mass, invariants, games, logic problems, pouring and weighting problems and amounts of information. Topics of the lessons are intentionally chosen so as not to rely upon previously learnt material; therefore, missing one lesson or several lessons does not hinder attendees from understanding ideas discussed at future lessons.

Most of the participants of Minor Mechmath are 11 to 14 years old, which corresponds to 5 th-8th grade. Work with this age category of children is organised as follows. Attendees of each grade are grouped into a socalled parallel. This means that all children are offered the same set of problems and the classes are conducted in several rooms (each room admits up to 30 attendees taught by $3-5$ tutors). Schoolchildren individually discuss their ideas and approaches to problems with the tutors. 
Thereby, schoolchildren learn to substantiate and defend their points of view while their arguments are being tested for consistency by the tutors. Also, they learn to look fairly at their mistakes and make due corrections. In turn, the form of a private dialogue allows the tutors to suggest hints and cast a light on possible routes to a solution.

An important form of popularisation activity at Minor Mechmath is a series of lectures in mathematics, mechanics and other related disciplines given by outstanding scientists who work at various departments of Moscow University. They present, in a comprehensible way, the most brilliant modern results and illustrate areas of actual scientific research.

Studying at Minor Mechmath effectively reveals the mathematical abilities of children and allows them to continue their education at specialised schools offering enhanced programmes in mathematics.

\section{Mechmath classes at Moscow School 54}

In 1988, the Department of Mechanics and Mathematics of Lomonosov Moscow State University launched a new form of educational activity. In collaboration with Secondary School 54 (Moscow), experienced scientists of the department started teaching schoolchildren an enhanced course of mathematics, physics and programming. The main idea was to raise the general level of Moscow schoolchildren who intended to continue their studies at Moscow University, to enlighten them on contemporary scientific achievements and involve them in actual research projects. The new educational approach was based on the experience of Minor Mechmath, Moscow Mathematical Olympiads and other intellectual competitions. Tutorials involved scientists of the department elaborating on fundamental school disciplines. The collaboration was backed by an agreement between the department and the school. The school formed new classes and the department selected candidates from all over Moscow.

The declared aim of the classes is a comprehensive development of each pupil's personality, a widening of the scope of their interests and an improving of their mathematical culture. The four-year course (8th-11th grade) empowers pupils and enables them to compete and win various top-level intellectual competitions, as well as successfully pass enrolment exams at all highrank universities.

The style of presentation and training of maths in the classes is highly original; it has nothing in common with conventional and routine teaching, which reduces to solving particular types of standard problem. The main tool of teaching is "the method of guided insights": the teachers offer a series of auxiliary problems, which guide the students and prepare them to unveil fundamental mathematical results themselves. Thereby, substantial mathematical facts appear to be discovered by children themselves rather than being obtruded by the teacher. This approach takes considerable time compared to the standard methods of teaching but it is worth the effort, as students gain indispensable experience of scientific research.
A distinctive feature of the academic programme practised in the classes is the idea of the educational spiral. According to this idea, fundamental concepts and facts appear throughout the entire course several times, each time at a higher level of comprehension. Owing to this approach, the students are permanently surrounded by all of the essential mathematical topics. This is in perfect agreement with recent achievements in the area of teenager psychology: a teenager as an individual is likely to put their surrounding reality to the test and to rediscover facts they are already aware of. And as soon as the facts have been re-established, they fit well into the pupil's current worldview.

The fact that the main disciplines in the classes are taught by actively working scientists encourages pupils to work hard and invest in mathematics. Moreover, children are offered additional, complicated research problems to work on, under the supervision of professional mathematicians. Every year, research projects are presented at the annual school conference, which gathers schoolchildren of all grades. Pupils present their projects to their schoolmates and teachers, thereby improving their presentation skills. In turn, the audience gets a chance to learn about many additional areas of elementary mathematics that usually fall outside the scope of the academic programme.

However, along with the attention given to teaching mathematics, physics and programming, the main educational efforts are focused on harmonious development of the children's personalities. Traditionally, schoolchildren go hiking and visit picturesque regions of the country. They also enjoy sightseeing in historical places of Russia and other European countries. Children go to theatres, exhibitions, museums and keep up with the cultural life of Moscow.

\section{References}

Web pages (in Russian)

of the Minor Department of Mechanics and Mathematics: http://mmmf.msu.ru of the School 54: http://www.moscowschool54.ru

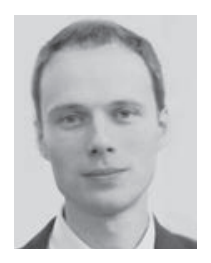

Alexander Vladimirovich Begunts [ab@] rector.msu.ru] is an associate professor at Lomonosov Moscow State University, where he teaches Calculus since 2002 and received his $P h D$ in mathematics in 2005. He was responsible for the admission process to the department and has worked with many high school students.

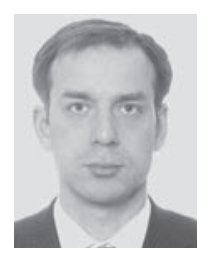

Anton Evgenievich Pankratiev [anton. pankratiev@gmail.com] is an associate professor at Lomonosov Moscow State University, where he received his $P h D$ in mathematics in 2001. The area of his interests includes algebra, computer science and information security. He is also involved in working with high school students and helping to organise various Olympiads. He is the head of "Malyi Mechmath". 